\title{
A Minimal Financial Market Model
}

\section{Eckhard Platen ${ }^{1}$}

September 29, 2000

\begin{abstract}
The paper proposes a financial market model that generates stochastic volatility and stochastic interest rate using a minimal number of factors that characterise the dynamics of the different denominations of the deflator. It models asset prices essentially as functionals of square root and Ornstein-Uhlenbeck processes. The resulting price processes exhibit stochastic volatility with leptokurtic log-return distributions that closely match those observed in reality. The resulting index of the market is negatively correlated with its volatility which models the wellknown leverage effect. The average growth rates of the different denominations of the deflator are Ornstein-Uhlenbeck processes which generates the typically observed long term Gaussianity of logreturns of asset prices.
\end{abstract}

1991 Mathematics Subject Classification: primary 90A12; secondary 60G30, 62P20. JEL Classification: G10, G13

Key words and phrases: stochastic volatility, financial market model, derivative pricing, square root process.

\footnotetext{
${ }^{1}$ University of Technology Sydney, School of Finance \& Economics and Department of Mathematical Sciences, PO Box 123, Broadway, NSW, 2007, Australia
} 


\section{Introduction}

An advanced financial market model should be analytically tractable and must reflect with a minimal number of factors essential stylised empirical facts. It must work equally well for derivative pricing and hedging as well as for risk measurement and portfolio management. All factors in such a market model should represent almost directly observable quantities.

The well-known Black-Scholes model (BSM) assumes that geometric Brownian motions generate the asset price dynamics. Although the theoretical and practical importance of the BSM cannot be underestimated, it is far from being satisfactory. The BSM has the drawback that it assumes deterministic volatility. This does not match historically observed stochastic volatility. For instance, in the context of option pricing practitioners have to correct for implied volatility skews and smiles due to stochastic volatility. The at the money short term implied volatility of an index has typically a strong negative correlation with the index itself which is also known as leverage effect. Furthermore, it has been observed that logreturns of asset prices over very long time periods tend to be Gaussian distributed. On the other hand, short term logreturns of asset prices have been shown to be leptokurtic with specific distributional properties. These stylised empirical facts should be well reflected in a sophisticated financial market model. Furthermore, such a model should acknowledge certain symmetries that naturally exist in a market as we will see below.

There is a rich literature on asset price modelling. For surveys on the modelling of stochastic volatility and other empirical stylised facts in the context of asset price dynamics, see Ghysels, Harvey \& Renault (1996) and Frey (1997).

A well studied group of asset price models covers the subordinated models, see, for instance, Clark (1973), Hurst, Platen \& Rachev (1997), Geman, Madan \& Yor (1998) and Heyde (1999) to mention just a few. These models use nondecreasing stochastic processes, usually with independent increments, to generate an independent stochastic operational time as directing process. A drawback for subordinated asset price models results from the fact that they typically assume independence between asset price and directing process which prevents these models to cover the above mentioned leverage effect.

An extensive literature exploits discrete time stochastic processes, for instance, $\mathrm{ARCH}$ and GARCH type asset price models. These models depend on a subjective choice of the time step size as an important parameter, see Engle \& Bollerslev (1986). Such a choice is not relevant in a stochastic differential equation framework, where drift and diffusion coefficient functions characterise the model.

Some authors suggest to include jumps into the asset price dynamics, see, e.g., Kou (2000). Unfortunately, it is almost impossible to identify objectively jumps in asset price data. Barndorff-Nielsen \& Shephard (1998) emphasise that a financial market model should be a continuous time model and use mean reverting Lévy 
processes to generate asset price dynamics which typically lead to incomplete markets. It is highly desirable that at least an idealised version of a financial market model forms a complete market. Dupire (1994) and Derman \& Kani (1994) developed volatility function approaches that lead to complete market models. These are in some sense generalisations of the constant elasticity of variance model, see, e.g., Cox \& Ross (1976). Unfortunately, as shown in Delbaen \& Shirakawa (1997), such models may, in general, not guarantee strict positivity of asset prices.

This paper proposes a diffusion model, the minimal market model (MMM), that uses a minimal number of almost directly observable factors, that are modelled as square root and Ornstein-Uhlenbeck processes under the real world probability measure. The basic building blocks of the proposed model are the different denominations of the deflator measured in units of primary assets. They determine all financial quantities including short rates, dividend rates, volatilities and risk premia. The MMM reflects well major stylised empirical facts as will be discussed at the end of the paper. Most importantly, it naturally generates stochastic volatility without using any additional stochastic volatility process. This is in contrast to most stochastic volatility models, see, for instance, Heston (1993) or Fouque, Papanicolau \& Sircar (1999), which use unobservable exogeneous processes to generate stochastic volatility. The MMM is computationally highly tractable and allows a good fit to historical and derivative data, as shown in Heath \& Platen (2000).

\section{Minimal Market Model}

\subsection{Savings Accounts and Deflator}

Let us define a primary asset as an income or loss producing asset, for instance, a stock currency or commodity. We consider in a market the evolution of the prices of $d+1$ primary assets, $d \in\{1,2, \ldots\}$, that is modelled on a filtered probability space $\left(\Omega, \mathcal{A}_{T}, \underline{\mathcal{A}}, P\right)$. Here the filtration $\underline{\mathcal{A}}=\left(\mathcal{A}_{t}\right)_{t \in[0, T]}$ fulfills the usual conditions with $\mathcal{A}_{0}$ being trivial, see Karatzas \& Shreve (1988). We assume that each primary asset has its own time value. The time value of the domestic currency is expressed via the corresponding savings account process $B^{0}=\left\{B^{0}(t), t \in[0, T]\right\}$, where

$$
B^{0}(t)=\exp \left\{\int_{0}^{t} f^{0}(s) d s\right\}
$$

for $t \in[0, T], T \in(0, \infty)$. This savings account accumulates continuously interest according to the domestic interest rate process $f^{0}=\left\{f^{0}(t), t \in[0, T]\right\}$ which describes the income rate from holding the domestic currency. The time value of the $j$ th primary asset is similarly modelled by the $j$ th savings account process 
$B^{j}=\left\{B^{j}(t), t \in[0, T]\right\}$, where

$$
d B^{j}(t)=B^{j}(t) f^{j}(t) d t
$$

for $t \in[0, T]$ with $B^{j}(0)=1$. The $j$ th income rate process $f^{j}=\left\{f^{j}(t), t \in[0, T]\right\}$ can be, for instance, a dividend rate, domestic or foreign interest rate or the net income rate from holding a commodity. In summary, the $j$ th savings account measures accumulated income or loss generated by the $j$ th asset in units of the $j$ th asset, $j \in\{0, \ldots, d\}$.

We assume that there exists a deflator, see Duffie (1996). This is a strictly positive $\underline{\mathcal{A}}$-adapted, continuous process that when used as numeraire makes any deflated price process an $(\underline{\mathcal{A}}, P)$-martingale. It is well-known that the deflator is the optimal growth portfolio as described in Karatzas \& Shreve (1998). Let us denote by $D^{j}=\left\{D^{j}(t), t \in[0, T]\right\}$ the $j$ th denomination of the deflator in units of the $j$ th primary asset $j \in\{0, \ldots, d\}$. Under the MMM the $j$ th denomination of the deflator $D^{j}(t)$ at time $t$ is specified as a functional of the form

$$
D^{j}(t)=\xi_{j}\left(Y^{j}(t)\right)^{q_{j}} G^{j}(t),
$$

where

$$
G^{j}(t)=\exp \left\{\int_{0}^{t} \eta^{j}(s) d s\right\}
$$

for $t \in[0, T]$ and $j \in\{0, \ldots, d\}$. The $j$ th initial parameter $\xi_{j}>0$ and the $j$ th exponent $q_{j} \in(0, \infty]$ are given constants. The $j$ th square root process $Y^{j}=\left\{Y^{j}(t), t \in[0, T]\right\}$, that appears in (2.2), is characterised by the stochastic differential equation (SDE)

$$
d Y^{j}(t)=\frac{\nu^{j}}{4} \varphi^{j}(t)\left(1-Y^{j}(t)\right) d t-\sum_{k=1}^{d} \gamma^{k, j}(t) \sqrt{Y^{j}(t)} d W^{k}(t)
$$

with $j$ th diffusion parameter $\varphi^{j}(t)=\sum_{k=1}^{d}\left(\gamma^{k, j}(t)\right)^{2}$ for $t \in[0, T]$ and initial value $Y^{j}(0)>0, j \in\{0, \ldots, d\}$.

Here $W^{1}, \ldots, W^{d}$ are independent standard Wiener processes. The $j$ th dimension $\nu^{j} \in\left(2+4 q_{j}, \infty\right)$ is a constant and the $k, j$ th volatility parameter $\gamma^{k, j}:[0, T] \rightarrow$ $(-\infty, \infty)$ is a deterministic function for $j \in\{0, \ldots, d\}, k \in\{1, \ldots, d\}$. Obviously, the square root process $Y^{j}$ fluctuates around its long term average value of one, where the diffusion parameter $\varphi^{j}$ controls the speed of its evolution and the dimension $\nu^{j}$ the magnitude of extreme fluctuations. For larger dimension $\nu^{j}$ extreme fluctuations are less likely.

The $j$ th growth rate process $\eta^{j}=\left\{\eta^{j}(t), t \in[0, T]\right\}$ governs according to $(2.2)$ the average growth of the $j$ th deflator. We assume that $\eta^{j}$ is an Ornstein-Uhlenbeck process with

$$
d \eta^{j}(t)=\alpha^{j}(t)\left(\bar{\eta}^{j}(t)-\eta^{j}(t)\right) d t+\sum_{k=1}^{d} \beta^{k, j}(t) d W^{k}(t)
$$


for $t \in[0, T]$ and initial value $\eta^{j}(0) \in(-\infty, \infty), j \in\{0, \ldots, d\}$. Here the $j$ th speed of adjustment parameter $\alpha^{j}:[0, T] \rightarrow(0, \infty), k, j$ th diffusion parameter $\beta^{k, j}:[0, T] \rightarrow(-\infty, \infty)$ and $j$ th average growth rate $\bar{\eta}^{j}:[0, T] \rightarrow(-\infty, \infty)$ are assumed to be given deterministic functions of time, $j \in\{0, \ldots, d\}, k \in$ $\{1, \ldots, d\}$.

Note that the SDEs for the square root and growth rate processes have unique, strong solutions with explicitly known transition densities, see Karatzas \& Shreve (1988).

\subsection{Asset Price Dynamics and Risk Premia}

The $j$ th deflated savings account process $\hat{S}^{j}=\left\{\hat{S}^{j}(t), t \in[0, T]\right\}$ is formed by the ratio

$$
\hat{S}^{j}(t)=\frac{B^{j}(t)}{D^{j}(t)}
$$

for $t \in[0, T]$ and $j \in\{0, \ldots, d\}$. Note the symmetry between deflated savings accounts in the model.

By application of the Itô formula, see Protter (1990), we obtain from (2.6), (2.1), (2.2) and (2.4) for the $j$ th deflated savings account the SDE

$$
\begin{aligned}
d \hat{S}^{j}(t)= & \hat{S}^{j}(t)\left[f^{j}(t)-\eta^{j}(t)+q_{j} \frac{\nu^{j}}{4} \varphi^{j}(t)+\frac{\varphi^{j}(t) q_{j}}{Y^{j}(t)}\left(\frac{q_{j}+1}{2}-\frac{\nu^{j}}{4}\right)\right] d t \\
& +\hat{S}^{j}(t) \sum_{k=1}^{d} \sigma^{k, j}(t) d W^{k}(t)
\end{aligned}
$$

for $t \in[0, T]$ with initial value

$$
\hat{S}^{j}(0)=\frac{1}{D^{j}(0)}
$$

and thus the $k, j$ th volatility of the $j$ th deflated savings account

$$
\sigma^{k, j}(t)=\frac{q_{j} \gamma^{k, j}(t)}{\sqrt{Y^{j}(t)}}
$$

for $j \in\{0, \ldots, d\}, k \in\{1, \ldots, d\}$ and $t \in[0, T]$.

By assumption the deflator makes all deflated price processes to martingales. This means $\hat{S}^{j}$ must to be a martingale and it follows from (2.7) that the $j$ th income rate fulfills the relation

$$
f^{j}(t)=\eta^{j}(t)+q_{j} \varphi^{j}(t)\left\{\frac{1}{Y^{j}(t)}\left[\frac{\nu^{j}}{4}-\frac{q_{j}+1}{2}\right]-\frac{\nu^{j}}{4}\right\}
$$


for $t \in[0, T]$ and $j \in\{0, \ldots, d\}$. Note, in the special case $\nu^{j}=2\left(q_{j}+1\right)$ the $j$ th income rate does not depend on the square root process $Y^{j}$.

From (2.6), (2.7) and (2.1) we obtain by application of the Itô formula the dynamics of the $j$ th deflator in the form

$$
\begin{aligned}
d D^{j}(t) & =d\left(\frac{B^{j}(t)}{\hat{S}^{j}(t)}\right) \\
& =D^{j}(t)\left[\left(f^{j}(t)+\sum_{k=1}^{d}\left(\sigma^{k, j}(t)\right)^{2}\right) d t-\sum_{k=1}^{d} \sigma^{k, j}(t) d W^{k}(t)\right]
\end{aligned}
$$

for $t \in[0, T]$ and $j \in\{0, \ldots, d\}$.

The exchange price $X^{i, j}(t)$ is the price of one unit of the $j$ th asset at time $t$ measured in units of the $i$ th asset. Using the $i$ th and $j$ th denominations of the deflator the $i, j$ th exchange price can be expressed as the ratio

$$
X^{i, j}(t)=\frac{D^{i}(t)}{D^{j}(t)}
$$

and it follows by application of the Itô formula and (2.11) that

$$
\begin{aligned}
d X^{i, j}(t)= & X^{i, j}(t)\left[\left(f^{i}(t)-f^{j}(t)\right) d t\right. \\
& \left.+\sum_{k=1}^{d}\left(\sigma^{k, j}(t)-\sigma^{k, i}(t)\right)\left\{-\sigma^{k, i}(t) d t+d W^{k}(t)\right\}\right]
\end{aligned}
$$

for $t \in[0, T]$ with initial value $X^{i, j}(0)=\frac{D^{i}(0)}{D^{j}(0)}$ and $i, j \in\{0, \ldots, d\}$.

Let us now consider the savings accounts which are essential for investment purposes. The $j$ th savings account price $S^{i, j}(t)$ at time $t$, when measured in units of the $i$ th primary asset, is given by the formula

$$
S^{i, j}(t)=X^{i, j}(t) B^{j}(t)
$$

and it follows by the Itô formula, (2.13) and (2.1) that

$$
d S^{i, j}(t)=S^{i, j}(t)\left[f^{i}(t) d t+\sum_{k=1}^{d}\left(\sigma^{k, j}(t)-\sigma^{k, i}(t)\right)\left\{-\sigma^{k, i}(t) d t+d W^{k}(t)\right\}\right]
$$

for $t \in[0, T]$ with $S^{i, j}(0)=X^{i, j}(0)$ and $i, j \in\{0, \ldots, d\}$.

Note in (2.15) that a premium appears in excess of the income rate of the denominating $i$ th asset. More precisely, the $i, j$ th risk premium has the form

$$
p^{i, j}(t)=\sum_{k=1}^{d}\left(\sigma^{k, i}(t)-\sigma^{k, j}(t)\right) \sigma^{k, i}(t)
$$


for $t \in[0, T]$ and $i, j \in\{0, \ldots, d\}$. For $i=0$ the equation (2.15) describes the dynamics of the $j$ th savings account expressed in the domestic currency.

\subsection{Contingent Claim Pricing}

We introduce the $(n+1)=4(d+1)$ factors $Z^{0}, \ldots, Z^{n}$ which are assumed to be given by the SDEs

$$
d Z^{\ell}(t)=\alpha^{\ell}\left(t, Z^{0}(t), \ldots, Z^{n}(t)\right) d t+\sum_{k=1}^{d} \beta^{\ell, k}\left(t, Z^{0}(t), \ldots, Z^{n}(t)\right) d W^{k}(t)
$$

for $t \in[0, T]$ with appropriate initial value $Z^{\ell}(0)$. The first $d+1$ factors are the square root processes, that is $Z^{\ell}(t)=Y^{\ell}(t)$, see $(2.4)$, for $t \in[0, T]$ and $\ell \in$ $\{0, \ldots, d\}$. The following $d+1$ factors play the role of the growth rate processes, that means $Z^{\ell+d+1}(t)=\eta^{\ell}(t)$, see $(2.5)$, for $t \in[0, T]$ and $\ell \in\{0, \ldots, d\}$. The third set of factors is chosen to generate the average growth of the denominations of the deflator, that is $d Z^{\ell+2 d+1}(t)=G^{\ell}(t)$, see $(2.3)$, for $t \in[0, T]$ and $\ell \in$ $\{0, \ldots, d\}$. The fourth group of factors represents the savings accounts, that is $Z^{\ell+3 d+2}(t)=B^{\ell}(t)$, see $(2.1)$, for $t \in[0, T]$ and $\ell \in\{0, \ldots, d\}$. In the standard version of the MMM, see Platen (2000), one only uses the first $d+1$ factors which are already sufficient to generate the key properties of the model, that is the stochastic volatility that generates the leverage effect and appropriate logreturn distributions.

We call a nonnegative deflated payoff $H=H\left(\bar{T}, Z^{0}(\bar{T}), \ldots, Z^{n}(\bar{T})\right) \in[0, \infty)$ that matures at $\bar{T} \in(0, T]$ a European contingent claim. One can show, see Platen (2000), that the deflated price $u_{H}\left(t, Z^{0}(t), \ldots, z^{n}(t)\right)$ at time $t \in[0, \bar{T}]$ of the contingent claim $H$ is given by the conditional expectation

$$
u_{H}\left(t, Z^{0}(t), \ldots, Z^{n}(t)\right)=E\left(H \mid \mathcal{A}_{t}\right) .
$$

Let us denote by $b(t)=\left[b^{k, j}(t)\right]_{k, j=1}^{d}$ the diffusion matrix of primary assets when denominated in domestic currency with $b^{k, j}(t)=\hat{S}^{j}(t)\left(\sigma^{j, k}(t)-\sigma^{0, k}(t)\right)$.

For given $H$ we introduce the vector $c(t)=\left(c^{1}(t), \ldots, c^{d}(t)\right)^{\top}$ with

$$
\begin{aligned}
c^{k}(t)= & \sum_{\ell=0}^{n} \beta^{\ell, k}\left(t, Z^{0}(t), \ldots, Z^{n}(t)\right) \frac{\partial u\left(t, Z^{0}(t), \ldots, Z^{n}(t)\right)}{\partial Z^{\ell}} \\
& -u\left(t, Z^{0}(t), \ldots, Z^{n}(t)\right) \sigma^{0, k}(t) .
\end{aligned}
$$

If the matrix $b(t)$ is nonsingular all $t \in[0, \bar{T}]$, then the number $\delta^{j}(t)$ of units of the $j$ th primary asset that must be held at time $t$ in a self-financing hedge portfolio that replicates $H$ is determined by the relations

$$
\left(\delta^{1}(t), \ldots, \delta^{d}(t)\right)^{\top}=b^{-1}(t) c(t)
$$


and

$$
\delta^{0}(t)=\frac{1}{\hat{S}^{0}(t)}\left(u\left(t, Z^{0}(t), \ldots, Z^{n}(t)\right)-\sum_{j=1}^{d} \delta^{j}(t) \hat{S}^{j}(t)\right) .
$$

To give an example for the price of a contingent claim it follows that a European call option with strike price $K$ and maturity $\bar{T}$ written on $D^{0}$ has in units of domestic currency the price

$$
C^{0}\left(t, Z^{0}(t), K, \bar{T}\right)=E\left(\frac{D^{0}(t)}{D^{0}(\bar{T})}\left(D^{0}(\bar{T})-K\right)^{+} \mid \mathcal{A}_{t}\right) .
$$

Since the deflated deflator has the constant value one the equations (2.19) - (2.21) determine with the specification $u\left(t, Z^{0}(t), \ldots, Z^{n}(t)\right)=1$ the number of units of primary assets that form the deflator.

\section{Properties of the MMM}

Let us finally discuss several properties of the MMM that are responsible for its simplicity and its good fit to empirical data. Most importantly, the MMM generates stochastic volatility without the use of an external volatility process. Note from (2.9) that the volatilities are proportional to the square root of the inverse of a square root process. This feature causes negative correlation between the $j$ th deflator and its volatility and thus generates the well observed leverage effect typically observed for indices. The resulting volatility process exhibits natural persistence of high or low values and generates threshold exceedances that are clustered and very similar to those observed in reality.

From (2.11) and (2.9) it follows that the log-return of the $j$ th deflator over a small time interval $[t, t+\varepsilon], \varepsilon>0$ has approximately the form

$$
\log \left(D^{j}(t+\varepsilon)\right)-\log \left(D^{j}(t)\right) \approx \frac{1}{\sqrt{Y^{j}(t)}} \sum_{k=1}^{d} \gamma^{j, k}(t) q_{j}\left(W^{k}(t+\varepsilon)-W^{k}(t)\right)
$$

which represents a Student $t$ distributed random variable since $Y^{j}(t)$ is gamma distributed. Thus log-returns over small time intervals of the $j$ th deflator are Student $t$ distributed under the MMM. This feature has been shown to be consistent with real data for most stock market indices in Hurst \& Platen (1997) and for index benchmarked stock prices in Platen (1999a).

Under the MMM the log-returns over very long time intervals of the $j$ th deflator are, in principle, Gaussian distributed due to the Gaussian growth rates in the MMM. This matches the widely observed Gaussianity of long term log-returns.

Since the leverage effect is well modelled by the MMM, implied volatility skews and smiles for European puts and calls on stock indices, stocks and currencies match those observed in practice, as shown in Heath \& Platen (2000). 
Under the MMM the short rate (2.10) is, in general, stochastic with properties that are similar to those actually observed, see Platen (1999b). For instance, the inverse of the $j$ th square root process generates a diffusion coefficient function with power 1.5 as observed in different empirical studies on short rates, see, e.g., Chan, Karolyi, Longstaff \& Sanders (1992).

The crucial advantages of the MMM are its good fit which generates only small changes in its few parameters and its high computational tractability. The key quantities for this complete model are almost directly observed. With a minimal number of factors the MMM overcomes many of the unrealistic features of the classical Black-Scholes model and most other models.

\section{References}

Barndorff-Nielsen, O. \& N. Shephard (1998). Aggregation and model construction for volatility models. University of Aarhus, CAF working paper No 10 .

Chan, K. C., G. A. Karolyi, F. A. Longstaff, \& A. B. Sanders (1992). An empirical comparison of alternative models of the short-term interest rate. J. Finance 47, 1209-1227.

Clark, P. K. (1973). A subordinated stochastic process model with finite variance for speculative prices. Econometrica 41, 135-159.

Cox, J. C. \& S. A. Ross (1976). The valuation of options for alternative stochastic processes. J. Financial Economics 3, 145-166.

Delbaen, F. \& H. Shirakawa (1997). Squared Bessel processes and their applications to the square root interest rate model. Preprint. Department of Industrial Engineering and Management, Tokio Institute of Technology.

Derman, E. \& I. Kani (1994). Riding on a smile. Risk 7, 32-39.

Duffie, D. (1996). Dynamic Asset Pricing Theory (2nd ed.). Princeton, University Press.

Dupire, B. (1994). Pricing with a smile. Risk 7, 18-20.

Engle, R. F. \& T. Bollerslev (1986). Modelling the persistence of conditional variances. With comments and a reply by the authors. Econometric Rev. 5(1), 1-87.

Fouque, J. P., G. Papanicolau, \& K. R. Sircar (1999). Financial modelling in a fast mean-reverting stochastic volatility environment. Asian-Pacific Financial Markets 6, 37-48.

Frey, R. (1997). Derivative asset analysis in models with level-dependent and stochastic volatility. Mathematics of Finance, Part II. CWI Quarterly 10(1), 1-34. 
Geman, H., D. Madan, \& M. Yor (1998). Asset prices are Brownian motion: only in business time. University Paris IX Dauphine, (working paper).

Ghysels, E., A. Harvey, \& E. Renault (1996). Stochastic volatility. In Statistical Methods in Finance, Volume 14 of Handbook of Statist., pp. 119-191. NorthHolland.

Heath, D. \& E. Platen (2000). Pricing and hedging of derivatives under an alternative asset price model with stochastic volatility. University of Technology Sydney, (working paper).

Heston, S. L. (1993). A closed-form solution for options with stochastic volatility with applications to bond and currency options. Rev. Financial Studies 6(2), 327-343.

Heyde, C. C. (1999). A risky asset model with strong dependence through fractal activity time. Columbia University, New York, (working paper).

Hurst, S. R. \& E. Platen (1997). The marginal distributions of returns and volatility. In Y. Dodge (Ed.), L $L_{1}$-Statistical Procedures and Related Topics, Volume 31 of IMS Lecture Notes - Monograph Series, pp. 301-314. Institute of Mathematical Statistics Hayward, California.

Hurst, S. R., E. Platen, \& S. T. Rachev (1997). Subordinated market index models: A comparison. Financial Engineering and the Japanese Markets 4, 97-124.

Karatzas, I. \& S. E. Shreve (1988). Brownian Motion and Stochastic Calculus. Springer.

Karatzas, I. \& S. E. Shreve (1998). Methods of Mathematical Finance, Volume 39 of Appl. Math. Springer.

Kou, S. G. (2000). A jump diffusion model for option pricing with three properties: Leptokurtic feature, volatility smile and analytical tractability. Columbia University, New York, (working paper).

Platen, E. (1999a). On the log-return distribution of index benchmarked share prices. Technical report, University of Technology, Sydney. QFRG Research Paper 22.

Platen, E. (1999b). A short term interest rate model. Finance and Stochastics 3(2), 215-225.

Platen, E. (2000). Risk premia and financial modelling without measure transformation. Technical report, Humboldt University Berlin. Research Report SFB 373.

Protter, P. (1990). Stochastic Integration and Differential Equations. Springer. 